\title{
A Lithium-Ion Capacitor Model working on a wide temperature range
}

\author{
S. Barcellona ${ }^{\text {a }}$ L. Piegari ${ }^{\text {b }}$ Senior Member, IEEE
}

\begin{abstract}
a Department of Electronics, Information and Bioengineering, Politecnico di Milano, Italy, simone.barcellona@polimi.it
${ }^{\mathrm{b}}$ Department of Electronics, Information and Bioengineering, Politecnico di Milano, Italy, luigi.piegari@polimi.it
\end{abstract}

CORRESPONDING AUTHOR: Simone Barcellona

e-mail: simone.barcellona@polimi.it

phone:+390223993758

\begin{abstract}
Energy storage systems are spreading both in stationary and transport applications. Among innovative storage devices, lithium ion capacitors (LiCs) are very interesting. They combine the advantages of both traditional electric double layer capacitors (EDLCs) and lithium ion batteries (LiBs). The behavior of this device is much more similar to ELDCs than to batteries. For this reason, several models developed for traditional ELDCs were extended to LiCs. Anyway, at low temperatures LiCs behavior is quite different from ELDCs and it is more similar to a LiB. Consequently, EDLC models works fine at room temperature but give worse results at low temperatures. This paper proposes a new electric model that, overcoming this issue, is a valid solution in a wide temperature range. Based on only five parameters, depending on polarization voltage and temperature, the proposed model is very simple to be implemented. Its accuracy is verified through experimental tests. From the reported results, it is also shown that, at very low temperatures, the dependence of the resistance from the current has to be taken into account.
\end{abstract}

Keywords—Lithium ion capacitors, Temperature dependence, Modeling, Current dependency

\section{Introduction}

In recent years, storage systems are spreading in many different applications ranging from low power/high energy to high power/low energy utilizations. Indeed, storages are, nowadays, a key element both for common devices, like mobiles, laptops and so on, and for particular applications such as electric vehicles and standalone PV systems [1]-[3]. Moreover, they are used to support and furnish ancillary services to smart grids [4]-[5]. Consequently, the requirements are day by day more stringent in terms of power density, energy density, lifetime and operating temperatures. In this scenario, new storage devices are proposed to fulfill some of the above-mentioned requirements. Among innovative storage devices, lithium ion capacitors (LiCs), are very interesting. They are hybrid energy storage systems between electric double layer capacitors (EDLCs) and lithium ion batteries (LiBs). In fact, 
the positive electrode is made from activated carbon and the negative one is composed of lithium ion pre-doped carbon. The introduction of this lithium ion electrode results in an operative voltage between 2.2 and $3.8 \mathrm{~V}$. This voltage range allows to achieve an increase in energy density by $40-70 \%$ in comparison with traditional EDLCs working between 0 and $2.7 \mathrm{~V}$. Indeed, traditional EDLCs are often used with a minimum voltage higher or equal to half of the maximum one because at lower voltages the current increases significantly to keep the power constant [6]-[8].

The main drawback of LiCs, if compared with EDLCs, lies in their higher sensibility to temperature variation. Their operative range temperature is reduced and their performances are more sensible to temperature changes [9]. This aspect will be deepened in Section 4.

The availability of a detailed model of storage systems is very useful to optimize the working operations of a given storage unit. For this reason, a wide modelling activity of storage systems has been proposed in literature in recent years [10]. Unfortunately, each device behaves differently from the others so, every time a new device is realized, new analyses to model it are necessary.

Some models of LiCs have been already proposed in literature [9], [11]-[13]. In [9] it is proposed a thermal and electric analysis of $\mathrm{LiC}$ cells. The variation of equivalent capacitance and internal resistance with the temperature is analyzed and also the variation of the parameters with the frequency is considered. Anyway, an electric equivalent circuit is not proposed and the variation of the capacitance with the operating voltage is neglected. In [11] the authors propose a simple model of LiCs based on a traditional model used for batteries and constituted by two RC branches. This model is not suitable to represent the fast dynamic of the device and does not take into account the temperature effect on electric parameters. In [12] an integrated electro-thermal model is proposed. The electrical model is composed by a voltage generator in series with an internal resistance and two parallel RC branches to take into account the dynamic answer of the device. Taking into account that, in their working principle, LiCs are similar to traditional EDLCs, in [13] the authors propose to extend a model valid from ELDCs to LiCs. The dynamic behavior is modeled with very high accuracy, but the model works well only at $30^{\circ} \mathrm{C}$. As it has been said, LiCs are much more sensible to temperature changes than EDLCs and therefore the model proposed in [13] does not work fine at temperatures lower than $30^{\circ} \mathrm{C}$. Electrochemical performance for different kind of LiCs was evaluated in [14].

A deep analysis of LiCs behavior over voltage and temperature range is proposed in [15]. In this paper the authors show how LiCs behave in very different ways at low $\left(-10^{\circ}-10^{\circ} \mathrm{C}\right)$ and normal $\left(10^{\circ}-50^{\circ} \mathrm{C}\right)$ temperatures. In order to catch both behaviors, different models are proposed for the two temperature ranges. In particular, a model with eleven parameters is proposed for the low temperature range. The model behaves well but the identification of eleven parameters can make difficult its use. In [16], a model with a reduced number of parameters (five) is proposed. Anyway, its accuracy in time domain is shown only at room temperature and, therefore, its usability seems limited as for the model proposed in [13]. 
In order to represent the dynamic behavior of LiCs under variable voltage in the range $2.2 \mathrm{~V}-3.8 \mathrm{~V}$ and changing temperatures between $-20^{\circ} \mathrm{C}$ and $60^{\circ} \mathrm{C}$, in this paper a modification of the circuital model of [13] is proposed. It will be shown that for a good representation of the low temperature behavior an additional RC branch is enough. The proposed model has only five parameters (as the model proposed in [16]) but its accuracy is comparable with the more complex model proposed in [15]. The parameters of the proposed model are tuned by means of electrochemical impedance spectroscopy (EIS) and interpolated to work at any voltage and any temperature. Finally, in order to verify the goodness of the proposed model, some experimental tests at different temperatures and voltages with step changes in the current have been performed. The results reported in the paper confirm that the proposed model allows to represent LiCs behavior in any working condition, both steady state and dynamics, with a reduced error.

\section{Frequency Domain Analysis}

LiCs, like others energy storage systems, show different behaviors at different frequency ranges. The problem is that the frequency response may depend on different parameters such as, polarization voltage, temperature, current and so on. The frequency response can be analyzed by the galvanostatic electrochemical impedance spectroscopy (GEIS). GEIS is a technique consisting in supplying the cell with a sinusoidal current with a fixed amplitude over a rated dc polarization voltage. The injected current has a fixed frequency and the resulting voltage is compared with the injected current. Through the FFT, the ratio between the fundamental harmonic of current and voltage is calculated obtaining the impendence of the cell at the fixed frequency. Moreover, it would be possible to add a dc current to the sinusoidal one in order to estimate the frequency response in function of the rated dc current, as well. This method has some limitations. Indeed, energy storage systems have a nonlinear behavior in function of the voltage. Hence, for high dc currents the consequent change in the polarization voltage makes the use of this technique impossible, otherwise, the estimated impedance loses sense. For this reason, one can use other kind of analysis, such as the time domain analysis, as proposed in [16]. In [13], the frequency domain analysis was used in order to estimate the behavior of LiCs under constant room temperature for different polarization voltages without regarding the current dependency. The results showed a very good match in time domain tests also with high currents. Consequently, we can state that, at least for this kind of energy storage systems under constant temperature, the current dependency can be neglected. The problem could be that for different temperatures the current dependency is much higher.

The aim of this work is to extend the model presented in [13] for a wide temperature range as far as it is possible to neglect the current dependency. Under this assumption, we have chosen to use the frequency domain analysis, without adding any dc current, to make the model proposed in [13] suitable to represent the behavior of the $\mathrm{LiC}$ also taking into account the temperature dependency without greatly increasing the complexity of the model. The first step is to verify if the addition of parameter 
temperature dependency in the model proposed in [13] is sufficient or not to represent the behavior of the LiC in a wide temperature range. Otherwise, we can adjust that model in order to extend the temperature range.

\section{Lithium Ion Capacitors Models}

The electric model proposed in [13] is shown in Fig. 1a. As previously mentioned, this model is able to represent LiCs behavior only at constant room temperature $\left(30^{\circ} \mathrm{C}\right)$ for different polarization voltages. In fact, the three parameters of that model, $R_{i}, C$ and $\tau$, are functions only of the dc polarization voltage $V$. This model is simple because it is characterized only by three parameters and temperature behavior is not taken into account. The equation underlying this model is the following:

$Z_{p}(j \omega, V)=R_{i}(V)+\frac{\tau(V) \operatorname{coth}(\sqrt{j \omega \tau(V)})}{C(V) \sqrt{j \omega \tau(V)}}=R_{p}(\omega, V)+\frac{1}{j \omega C_{p}(\omega, V)}$.

The resistor $R_{i}$ represents the ac resistance that, when a current step is imposed, causes the voltage drop proportional to the current step shown by the LiC. The second term of the (1) is the Warburg diffusion impedance which well represents the behavior for middle and low frequencies where the $\mathrm{LiC}$ is practically a pure capacitor. From a mathematical point of view, the inverse Laplace transformation of the Warburg term is very complicated to be solved. For this reason, this term could be simplified as reported in

[17]. This way, Warburg term can be modeled by an infinite series connection of RC branches as reported in Fig. 1. From a practical point of view, six branches are sufficient to approximate the Warburg term in a good way $(n=6)$. Anyway, whatever is the number of branches chosen for the model, the different parameters are always three.

Actually, at very low frequencies the LiC presents a not pure capacitor behavior because of very long time dynamics, such as recombination phenomena and self-discharge effect, that are neglected in this work. In other hands, in parallel to that model, some parallel RC branches, that take into account also these dynamics, could be added as reported in [18].

Fig. 2 shows spectroscopies in Nyquist diagrams for different polarization voltages and temperatures. Looking at these figures, we can recognize that for high temperatures $\left(30-60^{\circ} \mathrm{C}\right)$ the frequency responses are very similar to the ones of EDLCs relative to the porous nature of the positive activated carbon electrode [19]. Consequently, the model (1) could be able to represent the LiCs behavior in this temperature range. Instead, for low temperatures $\left(0-30^{\circ} \mathrm{C}\right)$ the frequency responses of $\mathrm{LiCs}$ are closer to the ones of lithium ion batteries (LiBs) relative to the negative lithium ion pre-doped carbon electrode [20]. This is a very interesting issue because in LiCs at low temperatures the so called charge transfer process (not present in EDLCs) is slower making arise the semicircle on the Nyquist plane typical of LiBs [15]. This means that for very low temperatures just adding the temperature dependency to model expressed by (1) is not sufficient to characterize the LiC behavior for all the temperatures. 
In order to overcome this issue, we added to model another RC branch with other two independent parameters. This way, we obtain a five parameters model in which all the parameters are depending by both the voltage and the temperature as reported in Fig. 1b. This novel model, is represented by equation (2)

$Z_{p}(j \omega, V, T)=R_{1}(V, T)+\frac{\tau_{1}(V, T) \operatorname{coth}\left(\sqrt{j \omega \tau_{1}(V, T)}\right)}{C_{1}(V, T) \sqrt{j \omega \tau_{1}(V, T)}}+\frac{R_{2}(V, T)}{1+j \omega R_{2}(V, T) C_{2}(V, T)}=R_{p}(\omega, V, T)+\frac{1}{j \omega C_{p}(\omega, V, T)}$.

The additional RC branch needs to represent the charge transfer impedance that arises at low temperatures. The obtained model is more complex than that presented in [13] because it has 5 parameters depending on voltage and temperature versus three parameters depending only on the voltage. On the contrary, it behaves much better at low temperatures. Moreover, the proposed model is simpler than the model proposed in [15]. In fact, the model reported in [15], has ten parameters in addition to the voltage source, resulting more complicated to be tuned and used.

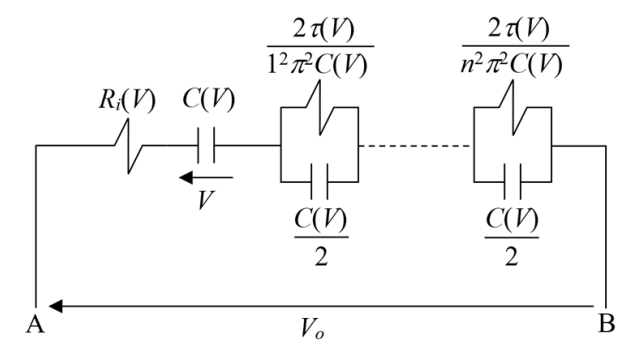

a)

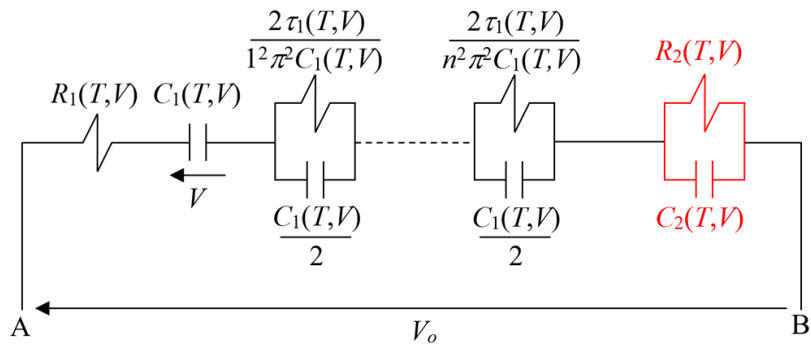

b)

Fig. 1. Equivalent circuit for $Z_{P}$, global impedance of $\mathrm{LiC}$ a) at fixed room temperature; b) in function of the temperature and de voltage. 

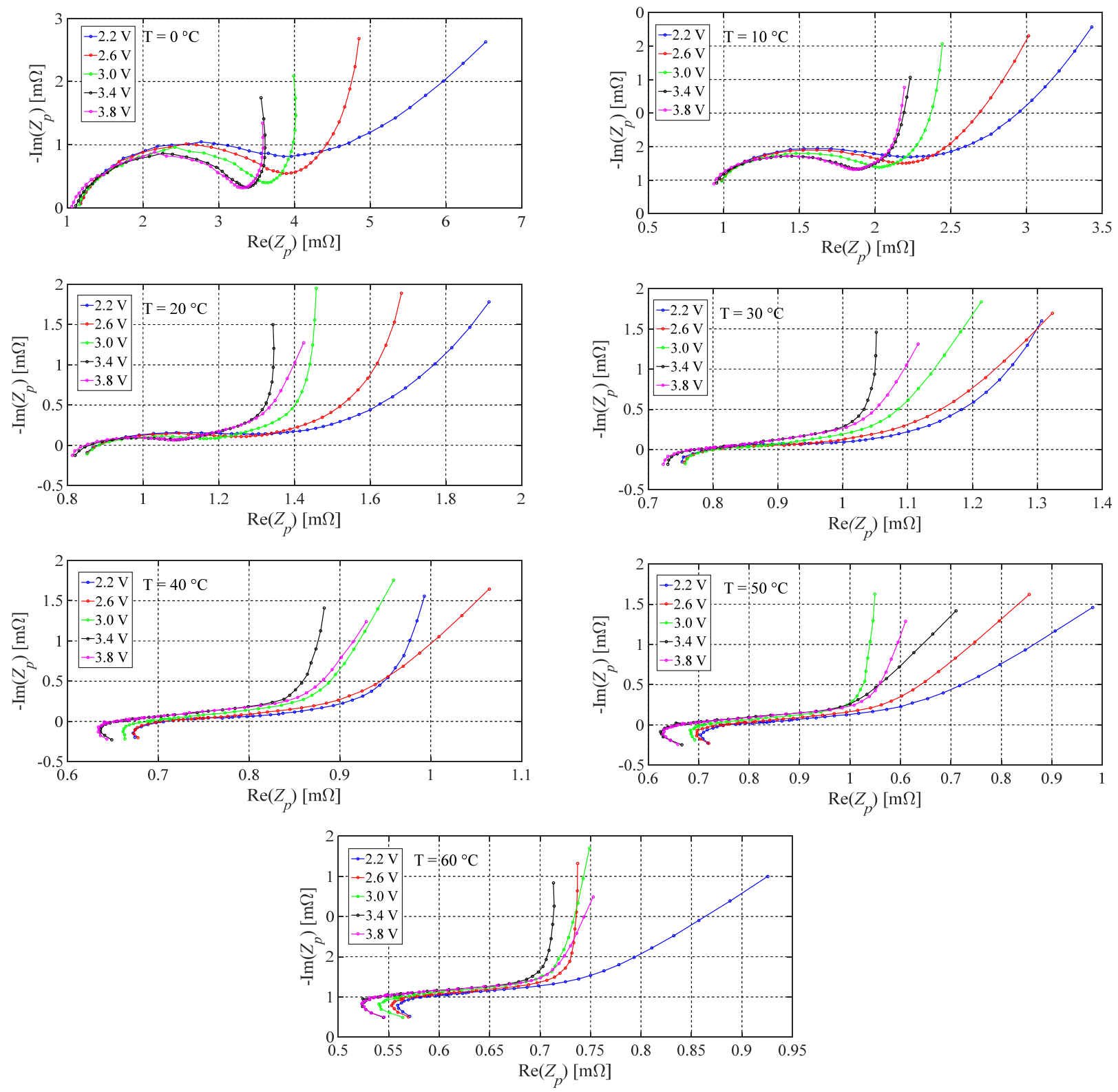

Fig. 2. Nyquist diagram for different polarization voltages and temperatures 


\section{Lithium Ion Capacitor Chemistry}

LiCs are classified as hybrid energy storage systems deriving from the combination of EDLCs and LiBs. In fact, they have the positive electrode made from activated carbon, as for EDLCs, while the negative electrode is composed of lithium ion pre-doped carbon, as for LiBs. In LiBs the energy is mainly stored in an electrochemical way, through the well-known faradaic reactions called ion intercalation/de-intercalation processes [21]. Conversely, in EDLCs, the energy is mainly stored in an electrostatic way, through the non-faradaic reactions due to the well-known double-layer capacitances. As a consequence, in LiCs both processes take place, on the respectively electrodes, and they are taken into account by the proposed novel model. In fact, the resistor $R_{1}$ represents the high frequency resistance due to the conductivity of electrodes, separator, and electrolyte. It corresponds to the intersection of the spectroscopy curve with the real axis on the Nyquist plane. The capacitor $C_{1}$ represents the main double layer capacitance and together with the time constant $\tau_{1}$ represent the ion mobility and the diffusion behavior due to the porous nature of the positive electrode, as for an EDLC. The resistor $R_{2}$ is the charge transfer resistance and together with $C_{2}$ represent the behavior of the charge transfer process related to ion intercalation/de-intercalation between the negative electrode and the electrolyte, as for a LiB.

At high temperatures, the high kinetic energy of lithium ions decreases the charge transfer resistance $R_{2}$ of the negative electrode. This involves that both the charge transfer and the diffusion processes are faster. Consequently, at the external terminals of the LiC the charge transfer process, related to the negative electrode, is not visible (the semicircle on the Nyquist plane tends to disappear) and the dominant effect is due to the positive electrode resulting in an overall equivalent EDLC behavior. Conversely, at low temperatures, the charge transfer resistance increases, making arise the semicircle on the Nyquist plane, whose behavior is similar to one of LiBs. For this reasons, it is used to state that LiCs act in the two different ways at high and low temperatures, respectively, as EDLC and $\mathrm{LiB}$, resulting much more sensible to temperature changes with respect to EDLCs.

\section{Parameter Identification}

Different typologies of LiCs, according to the configuration of the electrodes, are available in the market [15]. In this paper we focus on a particular laminar cell developed by JSR Micro (CLQ1100S1A). This cell is composed of a lithium ion pre-doped carbon electrode and an activated carbon electrode. In the author's opinion the proposed model may be tuned also for other kind of electrodes configurations. Further investigations would be necessary but this is out of the scope of this work.

One of these cells was tested at the Department of Electronics, Information and Bioengineering, Politecnico di Milano. The main goal of these tests is to verify the applicability of the proposed model where the parameters have to be estimated by analyzing their dependence on polarization voltage and temperature. In order to meet this goal, the cell was tested in a frequency range of $100 \mathrm{mHz}$ up to $100 \mathrm{~Hz}$ using GEIS method, as done in [13]-[16] and [18]. Performing GEIS the impedance spectroscopy is 
obtained, i.e. the frequency response is achieved. The equipment used in order to get the frequency analysis consisted of a 100-A booster (VMP3B-100) connected to a potentiostat (SP-150) from Biologic Science Instruments, controlled by a PC via USB with EC-LAB software.

In order to verify the proposed model, the tests were repeated at different temperatures, using a climate chamber, and at different polarization voltages. The temperature range is between $0{ }^{\circ} \mathrm{C}$ and $60^{\circ} \mathrm{C}$, with a step of $10^{\circ} \mathrm{C}$. The polarization voltage range is between $2.2 \mathrm{~V}$ and $3.8 \mathrm{~V}$ with a step of $0.4 \mathrm{~V}$. Therefore, thirty-five different spectroscopies tests were performed. For each polarization voltage and temperature, the five parameters of the model were estimated using the complex least-square minimization method applied to (2). In particular, in order to minimize the error of a complex quantity, the total vector error was used and applied to the following function:

$\varepsilon=\sqrt{\left(\frac{\left\|\boldsymbol{R}_{p}-\boldsymbol{R}_{p}^{*}\right\|}{\left\|\boldsymbol{R}_{p}^{*}\right\|}\right)^{2}+\left(\frac{\left\|\boldsymbol{C}_{p}-\boldsymbol{C}_{p}^{*}\right\|}{\left\|\boldsymbol{C}_{p}^{*}\right\|}\right)^{2}}$

where $\boldsymbol{R}_{p}{ }^{*}$ and $\boldsymbol{C}_{p}{ }^{*}$ are obtained by the experimental measurements of the impedance, while $\boldsymbol{R}_{p}$ and $\boldsymbol{C}_{p}$ are the relative vectors of values obtained using eq. (2). The procedure of the minimization has been applied to evaluate $R_{1}, C_{1}, R_{2}, C_{2}$, and $\tau_{1}$; the resulting parameters are reported in Table 1.

Fig. 3 shows the comparison of the resistances $R_{p}$ and capacitances $C_{p}$ versus frequency obtained using the model (2) (continuous line) and the experimental data (marked points) at different temperatures and dc polarization voltages. Looking at the behaviours of resistances and capacitances versus frequency, it is possible to recognize a rather well agreement between the experimental data and the fitting of model (2).

\begin{tabular}{|c|c|c|c|c|c|c|}
\hline $\mathbf{V}[\mathbf{V}]$ & $\left.\mathbf{T}{ }^{\circ} \mathbf{C}\right]$ & $\mathbf{R}_{1}[\mathbf{m} \Omega]$ & $\mathbf{C}_{1}[\mathbf{F}]$ & $\tau_{1}[\mathbf{s}]$ & $\mathbf{R}_{2}[\mathbf{m} \Omega]$ & $\mathbf{C}_{2}[\mathbf{F}]$ \\
\hline \multirow{7}{*}{2.2} & 0 & 2.113 & 741.8 & 5.137 & 1.698 & 5.140 \\
\cline { 2 - 7 } & 10 & 1.450 & 701.7 & 2.342 & 0.6110 & 5.464 \\
\cline { 2 - 7 } & 20 & 1.018 & 852.6 & 1.353 & 0.2033 & 7.863 \\
\cline { 2 - 7 } & 30 & 0.8061 & 936.9 & 0.9062 & 0.08789 & 9.187 \\
\cline { 2 - 7 } & 40 & 0.7203 & 965.0 & 0.6891 & 0 & 413.6 \\
\cline { 2 - 7 } & 50 & 0.6436 & 1059 & 0.6229 & 0 & 614.2 \\
\cline { 2 - 7 } & 60 & 0.5952 & 1035 & 0.5339 & 0 & 433.2 \\
\hline \multirow{6}{*}{2.6} & 0 & 1.772 & 591.7 & 2.100 & 1.703 & 2.586 \\
\cline { 2 - 7 } & 10 & 1.419 & 711.9 & 1.452 & 0.5913 & 4.145 \\
\cline { 2 - 7 } & 20 & 1.034 & 798.6 & 0.9041 & 0.1524 & 8.196 \\
\cline { 2 - 7 } & 30 & 0.8313 & 898.2 & 0.7790 & 0.04194 & 16.21 \\
\cline { 2 - 7 } & 40 & 0.7106 & 930.3 & 0.6458 & 0 & 567.7 \\
\cline { 2 - 7 } & 50 & 0.6143 & 953.2 & 0.5718 & 0 & 669.6 \\
\hline
\end{tabular}




\begin{tabular}{|c|c|c|c|c|c|c|}
\hline & 60 & 0.5531 & 921.4 & 0.4693 & 0 & 564.0 \\
\hline \multirow{7}{*}{3.0} & 0 & 1.632 & 739.0 & 1.771 & 1.615 & 2.278 \\
\hline & 10 & 1.346 & 750.8 & 1.007 & 0.5351 & 3.879 \\
\hline & 20 & 0.9432 & 787.2 & 0.7175 & 0.1610 & 3.832 \\
\hline & 30 & 0.8334 & 841.1 & 0.6420 & 0 & 632.3 \\
\hline & 40 & 0.6654 & 878.2 & 0.5919 & 0 & 643.7 \\
\hline & 50 & 0.5738 & 844.2 & 0.4763 & 0 & 723.8 \\
\hline & 60 & 0.5360 & 844.7 & 0.4483 & 0 & 494.9 \\
\hline \multirow{7}{*}{3.4} & 0 & 1.558 & 900.7 & 1.408 & 1.523 & 2.723 \\
\hline & 10 & 1.238 & 1012 & 1.222 & 0.5131 & 3.954 \\
\hline & 20 & 0.8404 & 1026 & 0.9121 & 0.1872 & 2.114 \\
\hline & 30 & 0.7724 & 1059 & 0.8164 & 0 & 585.1 \\
\hline & 40 & 0.6275 & 1100 & 0.7618 & 0 & 714.3 \\
\hline & 50 & 0.5615 & 1111 & 0.6965 & 0 & 633.1 \\
\hline & 60 & 0.5153 & 1096 & 0.6154 & 0 & 741.3 \\
\hline \multirow{7}{*}{3.8} & 0 & 1.476 & 1188 & 2.690 & 1.542 & 2.180 \\
\hline & 10 & 1.222 & 1131 & 4.143 & 0.5216 & 1.277 \\
\hline & 20 & 0.8903 & 1222 & 4.435 & 0.1371 & 1.167 \\
\hline & 30 & 0.7770 & 1194 & 882.2 & 0 & 0.9517 \\
\hline & 40 & 0.6307 & 1262 & 683.9 & 0 & 0.9222 \\
\hline & 50 & 0.5569 & 1221 & 697.0 & 0 & 0.7910 \\
\hline & 60 & 0.5177 & 1259 & 626.3 & 0 & 0.7590 \\
\hline
\end{tabular}



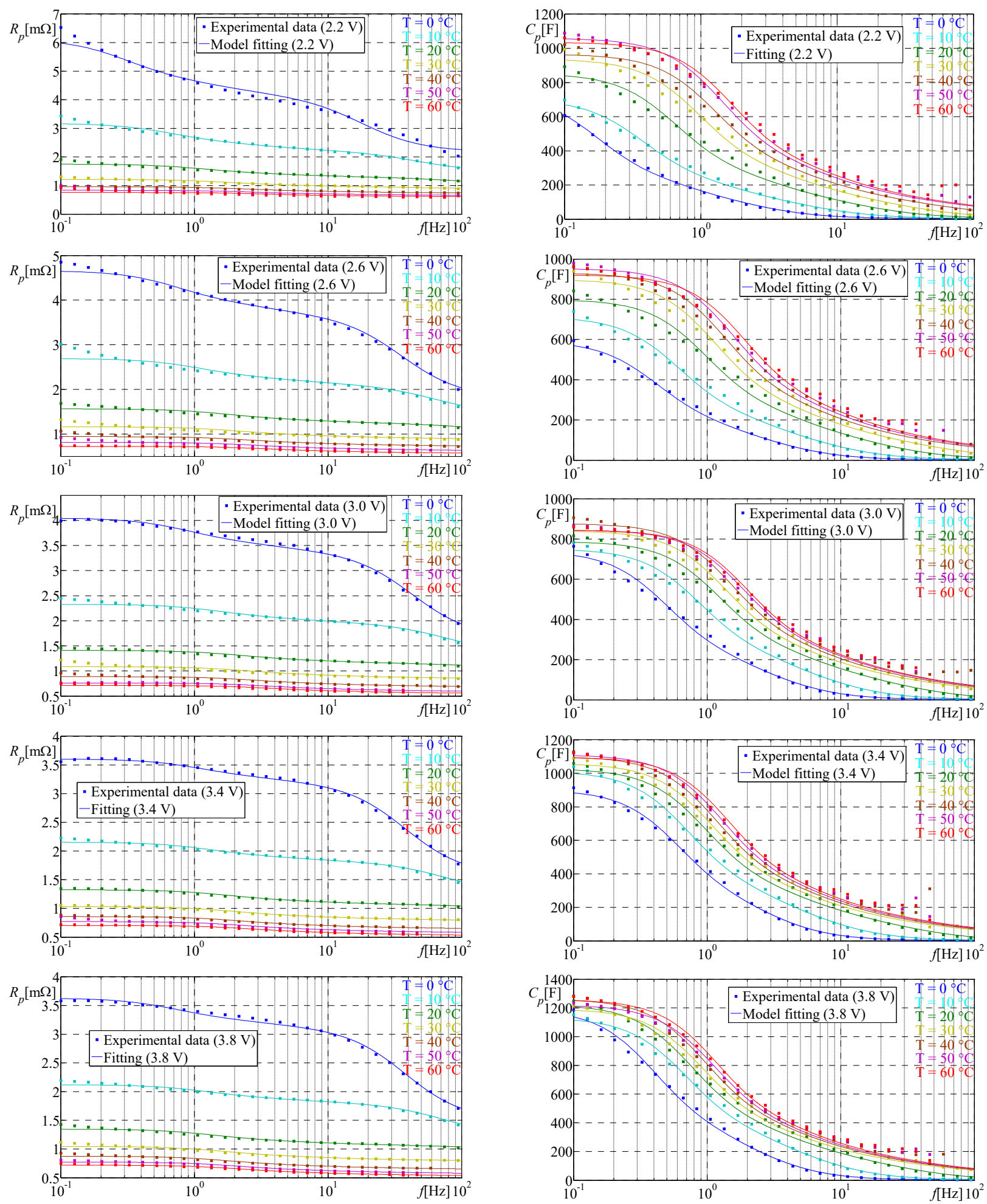

Fig. 3. CLQ1100S1A. Model-based fitting (continuous line) and experimental data (marked point), for different polarization voltages at different temperatures.

For both resistances and capacitance trends, it is possible to see that, in the most cases, at low frequencies the experimental data tends to be a little higher than the fitting model data. This fact is due to recombination phenomena [18], that are not taken into account by the proposed model, as previously mentioned in Section 3. Indeed, the present paper aims to find a model that works 
in a frequency range of $100 \mathrm{mHz}-100 \mathrm{~Hz}$, therefore, this parallel branch is avoided. Moreover, it is possible to note that at high frequencies some capacitance trends present a mismatch with respect to the related fitting curve. The deviation at high frequency can be explained taking into account the inductance of the measurement loop. Once the five parameters are estimated, another interpolation is requested. This time, the interpolation is made using the real least-square minimization method with polynomial surfaces as functions of both polarization voltage and temperature.

In Fig. 4 the values of the five parameters, as functions of temperature and voltage are reported with their related fitting curves.
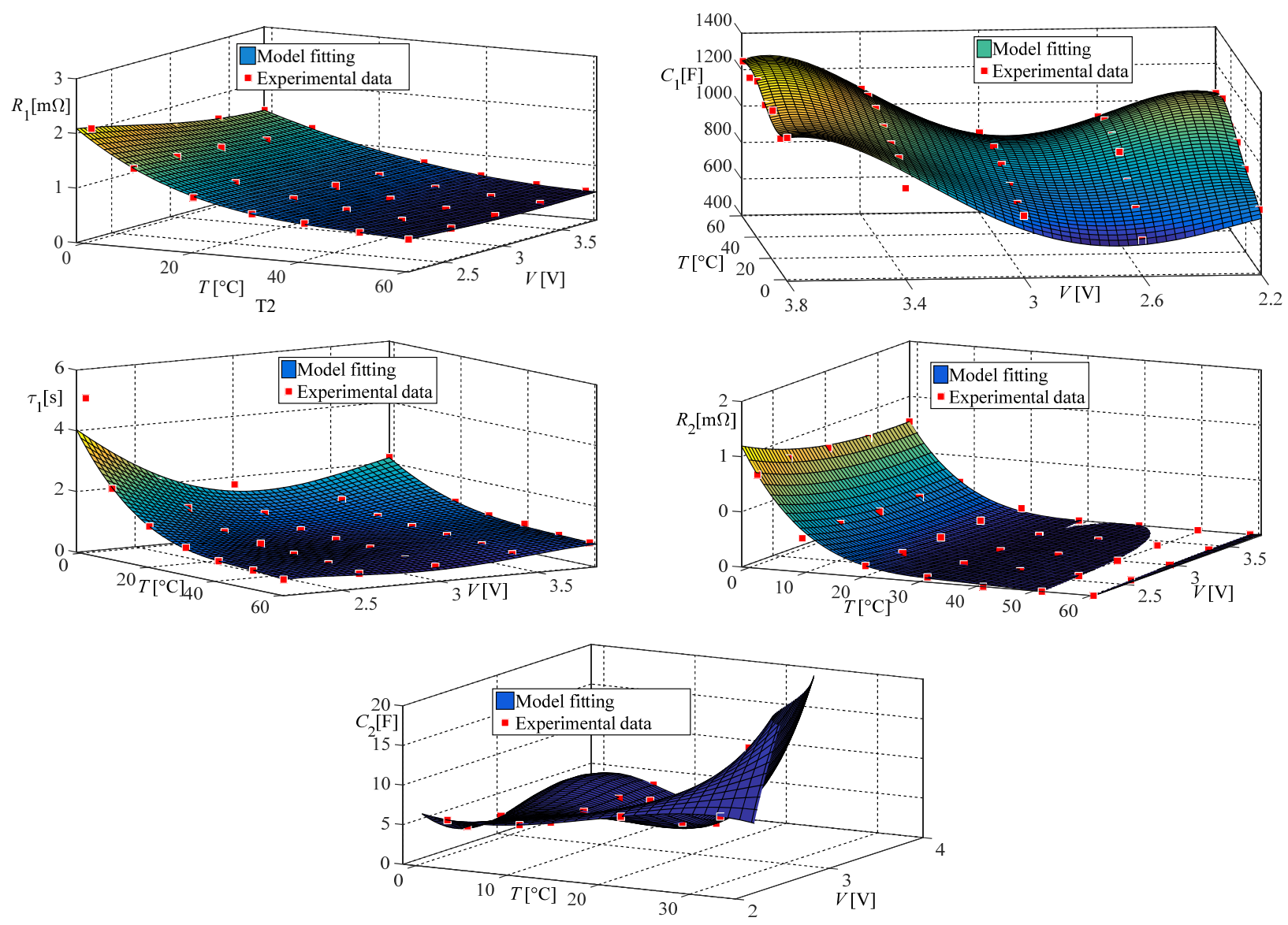

Fig. 4. Parameters $R_{1}, C_{1}, \tau_{1}, R_{2}, C_{2}$ : model-based fitting (continuous line) and experimental data (marked line)

In order to be general and synthetic, the five polynomial functions are as follows

$$
\begin{aligned}
& X(T, V)=x_{p 00}+x_{p 10} T+x_{p 01} V+x_{p 20} T^{2}+x_{p 11} T V+ \\
& +x_{p 02} V^{2}+x_{p 30} T^{3}+x_{p 21} T^{2} V+x_{p 12} T V^{2}+x_{p 03} V^{3}+ \\
& +x_{p 40} T^{4}+x_{p 31} T^{3} V+x_{p 22} T^{2} V^{2}+x_{p 13} T V^{3}+x_{p 04} V^{4} \\
& +x_{p 50} T^{5}+x_{p 41} T^{4} V+x_{p 32} T^{3} V^{2}+x_{p 23} T^{2} V^{3}+x_{p 14} T V^{4} .
\end{aligned}
$$


where $X(T, V)$ is each one of the five parameters. The values of the polynomial coefficients are reported in Table 2. Anyway, for each parameter, a different polynomial function degree can be necessary. For this reason in Table 2 some cells are empty because they refer to terms of (4) not present for the parameter of that column.

In particular, $R_{1}, C_{2}$ and $\tau_{1}$ can be approximated by a $4^{\text {th }}$ degree polynomial surface with 3 degrees for the temperature and 4 degrees for dc voltage. $C_{1}$ can be approximated by a $5^{\text {th }}$ degree polynomial surface with 5 degrees for the temperature and 4 degrees for dc voltage. $R_{2}$ can be approximated by a $4^{\text {th }}$ degree polynomial surface with 4 degrees for the temperature and 4 degrees for dc voltage.

The parameter $C_{2}$ was interpolated using only the values corresponding to not nil values of $R_{2}$. Indeed, from Table 2 , it is possible to note that, starting from $40{ }^{\circ} \mathrm{C}$ corresponding to a polarization voltage of $2.2 \mathrm{~V}$ and $2.6 \mathrm{~V}$, and starting from $30{ }^{\circ} \mathrm{C}$ corresponding to a polarization voltage of $3 \mathrm{~V}, 3.4 \mathrm{~V}$ and $3.8 \mathrm{~V}$, the resistance $R_{2}$ is nil. This fact means that starting from a temperature about $30-40{ }^{\circ} \mathrm{C}$, the $R_{2} C_{2}$ branch, added to the model of Fig. 1, becomes a short circuit. This confirms that the new $R_{2} C_{2}$ branch is necessary to model the behavior of the LiC cell at low temperatures. In fact, the model proposed in [13] without the $R_{2} C_{2}$ branch was proposed only for $30^{\circ} \mathrm{C}$ operations. Table 2 shows the coefficients of the polynomial functions.

Definitely, the procedure to obtain the five parameters, $R_{1}, C_{1}, R_{2}, C_{2}$, and $\tau_{1}$, as functions of temperature and dc voltage is the following: the first step is to interpolate the model (2) through the use of experimental data of resistance and capacitance versus frequency trends. This is made minimizing the total vector error (3). By this step, the five parameters $R_{1}, C_{1}, R_{2}, C_{2}$, and $\tau_{1}$ are obtained for each temperature and dc polarization voltage (Table 1). After that, these five parameters are interpolated using the least-square minimization method with polynomial surfaces. By this second step, the five functions related to the five parameters (4) are obtained.

\begin{tabular}{|l|c|c|c|c|c|}
\hline & $R_{1}$ & $C_{1}$ & $\tau_{1}$ & $R_{2}$ & $C_{2}$ \\
\hline$x_{p 00}$ & -0.001979 & -31210 & -11.38 & 0.006862 & -86.67 \\
\hline$x_{p 10}$ & -0.0001278 & -1299 & -1.323 & -0.0003552 & -24.7 \\
\hline$x_{p 01}$ & 0.005916 & 49630 & 40.15 & -0.0038 & 192.2 \\
\hline$x_{p 20}$ & $3.296 \cdot 10^{-6}$ & 7.604 & 0.01526 & $8.394 \cdot 10^{-6}$ & 0.4427 \\
\hline$x_{p 11}$ & $1.422 \cdot 10^{-5}$ & 1623 & 0.8482 & 0.0001236 & 23.92 \\
\hline$x_{p 02}$ & -0.002953 & -28110 & -28.12 & 0.0008335 & -130.3 \\
\hline$x_{p 30}$ & $-1.84 \cdot 10^{-8}$ & 0.01184 & $-4.745 \cdot 10^{-5}$ & $-1.093 \cdot 10^{-7}$ & -0.008089 \\
\hline$x_{p 21}$ & $-1.134 \cdot 10^{-6}$ & -7.786 & -0.007489 & $-1.193 \cdot 10^{-6}$ & -0.245 \\
\hline$x_{p 12}$ & $9.594 \cdot 10^{-6}$ & -735.6 & -0.1686 & $-2.169 \cdot 10^{-5}$ & -7.544 \\
\hline$x_{p 03}$ & 0.0005790 & 6863 & 7.345 & $-5.585 \cdot 10^{-5}$ & 35.72 \\
\hline$x_{p 40}$ & - & -0.0006485 & - & $5.965 \cdot 10^{-10}$ & - \\
\hline
\end{tabular}




\begin{tabular}{|c|c|c|c|c|c|}
\hline$x_{p 31}$ & $4.729 \cdot 10^{-9}$ & 0.01838 & $1.023 \cdot 10^{-5}$ & $3.581 \cdot 10^{-9}$ & 0.003471 \\
\hline$x_{p 22}$ & $9.067 \cdot 10^{-8}$ & 2.247 & 0.001005 & $1.206 \cdot 10^{-7}$ & 0.02473 \\
\hline$x_{p 13}$ & $-1.752 \cdot 10^{-6}$ & 146 & 0.008467 & $1.023 \cdot 10^{-6}$ & 0.7924 \\
\hline$x_{p 04}$ & $-3.934 \cdot 10^{-5}$ & -608.1 & -0.6530 & $-2.845 \cdot 10^{-7}$ & -3.448 \\
\hline$x_{p 50}$ & - & $2.641 \cdot 10^{-6}$ & - & - & - \\
\hline$x_{p 41}$ & - & $6.803 \cdot 10^{-5}$ & - & - & - \\
\hline$x_{p 32}$ & - & -0.004592 & - & - & - \\
\hline$x_{p 23}$ & - & -0.1903 & - & - & - \\
\hline$x_{p 14}$ & - & -10.86 & - & - & - \\
\hline
\end{tabular}

Table 2. POLYNOMIAL COEFFICIENTS OF THE PARAMETERS

\section{Experimental Tests}

In order to verify the proposed model over a wide frequency range, at different temperatures and polarization voltages, some current steps were injected in the LiC cell using the same instrumentations used for the GEIS. The same current profile was injected in the simulation model as well. In particular, the sequence of negative and positive current steps from 40 to $100 \mathrm{~A}$ injected in the LiC cell is shown in Fig. 5. This current profile is done with high current steps being the maximum cell current equal to $100 \mathrm{~A}$.

Simulations were carried out using Simulink/Matlab. For each temperature between 0 and $60^{\circ} \mathrm{C}$ with a step of $10^{\circ} \mathrm{C}$, three different tests were made. These three tests correspond to polarization voltage values equal to $2.6 \mathrm{~V}, 3 \mathrm{~V}$ and $3.4 \mathrm{~V}$. In total twentyone different tests were done. For sake of brevity, only tests at polarization voltage of $3.0 \mathrm{~V}$ are shown. The choice to show these tests is because among all, they represent the intermediate case between the worst and the best results. Anyway, the accuracy of the model for all tests is achievable from Table 3 in which the resulting coefficient of determination are reported.

Fig. 5 shows the comparison of the output voltage trends of experimental data (red line) and the simulated ones using both the model (2) (blue line) and (1) (green line), for some of the experimental tests.

Looking at these figures, it is possible to recognize a good agreement between the experimental data (red line) and the simulated one using the model (2) (blue line), for every temperature and polarization voltage. On the other hand, in particular for low temperatures, the agreement of the experimental data and the simulated one through the use of the model (1) is much less accurate than the model (2). Only for temperatures, close to $30^{\circ} \mathrm{C}$ the agreement of the model (1) is good.

Table III shows the coefficient of determination, denoted by $R^{2}$, of both model (1) and (2), for each test. The coefficient of determination is defined as the measure of how well a fitting curve represents the data. If the fitting curve passes exactly through each data point $R^{2}$ is equal to 1 and the fit is perfect. As much the line is away from the points, the less it is able to represent it. The minimum $R^{2}$ value of 0 indicates that the curve does not fit the data at all. The mathematical definitions is the following 
$R^{2}=1-\frac{\sum\left(V_{o}-V_{o}^{*}\right)^{2}}{\sum\left(V_{o}-V_{o M}\right)^{2}}$

where $V_{o}$ is the experimental output voltage in a certain time instant, $V_{o}^{*}$ is the modeled output voltage in the same time instant and $V_{o M}$ is the mean value of the experimental data. In this way, it is possible to obtain, during a period of observation, an index of how is much or less good the model with respect to the experimental data over the whole period.

The $R^{2}$ of the model (2) is between 0.9654 and 0.9978 . The $R^{2}$ of the model (1) is between 0.5392 and 0.9954 .

\begin{tabular}{|c|c|c|c|}
\hline $\mathbf{V}_{\mathrm{dc}}[\mathbf{V}]$ & $\mathbf{T}\left[{ }^{\circ} \mathbf{C}\right]$ & $R^{2}$ of model (2) & $R^{2}$ of model (1) \\
\hline \multirow{7}{*}{2.6} & 0 & 0.9674 & 0.5393 \\
\hline & 10 & 0.9907 & 0.7671 \\
\hline & 20 & 0.9846 & 0.9597 \\
\hline & 30 & 0.9654 & 0.9754 \\
\hline & 40 & 0.9933 & 0.9728 \\
\hline & 50 & 0.9934 & 0.9217 \\
\hline & 60 & 0.9837 & 0.8755 \\
\hline \multirow{7}{*}{3.0} & 0 & 0.9767 & 0.5577 \\
\hline & 10 & 0.9973 & 0.7919 \\
\hline & 20 & 0.9921 & 0.9674 \\
\hline & 30 & 0.9927 & 0.9954 \\
\hline & 40 & 0.9956 & 0.9822 \\
\hline & 50 & 0.9955 & 0.9482 \\
\hline & 60 & 0.9899 & 0.9178 \\
\hline \multirow{7}{*}{3.4} & 0 & 0.9759 & 0.6070 \\
\hline & 10 & 0.9978 & 0.8051 \\
\hline & 20 & 0.9952 & 0.9701 \\
\hline & 30 & 0.9931 & 0.9938 \\
\hline & 40 & 0.9939 & 0.9818 \\
\hline & 50 & 0.9954 & 0.9516 \\
\hline & 60 & 0.9923 & 0.9168 \\
\hline
\end{tabular}

Table 3. COEFFICIENTS OF DETERMINATION $\left(\mathrm{R}^{2}\right)$

From Table 3 it is possible to see that the $R^{2}$ is depending on both the dc polarization voltage and temperature. In the worst case, the minimum $R^{2}$ of model (2) is equal to 0.9654 at $30{ }^{\circ} \mathrm{C}$ and $2.6 \mathrm{~V}$. In all other cases, it is bigger than 0.97 . Moreover, the agreement between the experiment data and the proposed model could be further enhanced by increasing the number of tests at different temperatures and polarization voltages, in order to obtain better interpolations. In fact, in the procedure to estimate the model parameters, there are two different interpolation sets. In order to improve the behavior of the proposed model, it would be 
better to increase the step of the temperature and dc polarization voltage for the second interpolation set. As consequence, the experimental data would be a lot and time to elaborate them would result expensive.
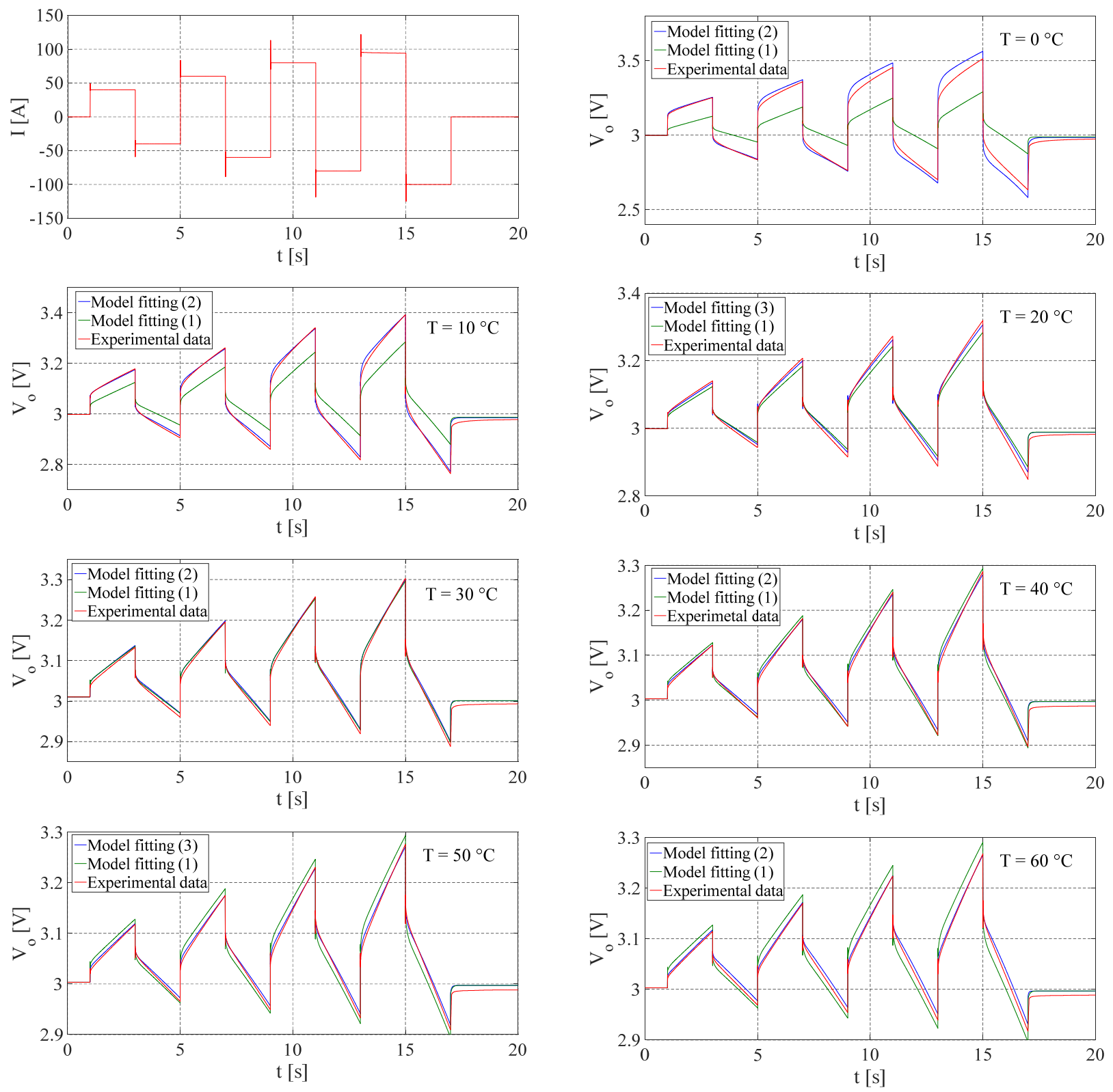

Fig. 5. Current profile and comparison between experimental and simulation tests at $3.0 \mathrm{~V}$ for different temperatures

\section{Current Dependency}

In the proposed model, the current dependency was not taken into account in order to verify as far as the current effect is negligible. Looking at Fig. 5 , at $0{ }^{\circ} \mathrm{C}$ for high currents, the proposed model presents a mismatch between experimental data and simulated one. The more is the current the more is the mismatch. This fact leads to think that this effect is due to current effect that at low temperatures arises. On the contrary, this effect disappears for high temperatures. 
In order to deepen this effect, other tests were done at very low temperatures. Fig. 6 shows the spectroscopies at -10 and -20 ${ }^{\circ} \mathrm{C}$. Looking at these figures, it is possible to recognize that the charge transfer impedance is much accentuated. At these very low temperatures, the $\mathrm{LiC}$ behavior is much more similar to the one of LiB. This confirms again that different currents can affect the LiC behavior.
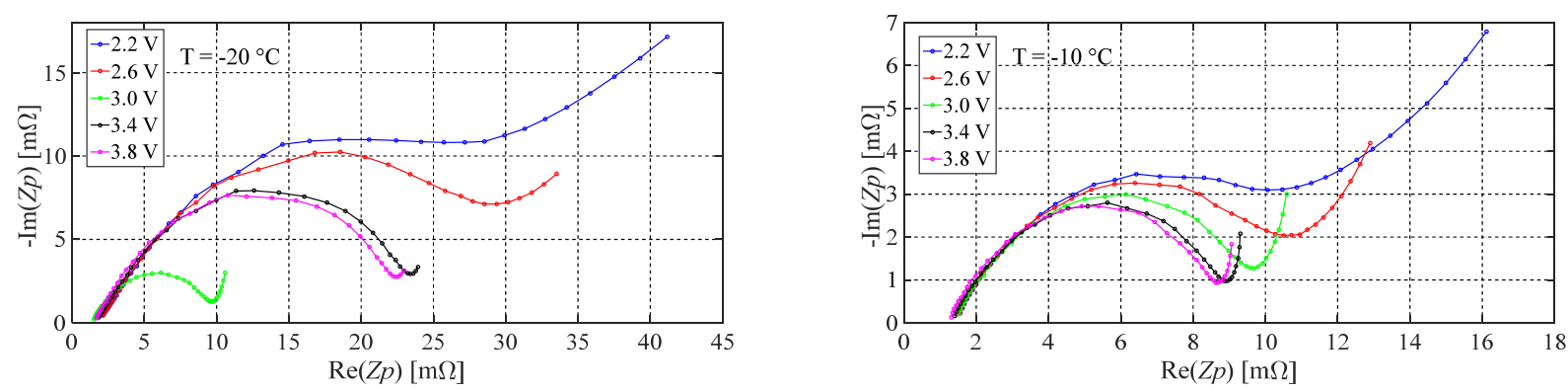

Fig. 6. Nyquist diagram for different polarization voltage at $-20{ }^{\circ} \mathrm{C}$ and $-10{ }^{\circ} \mathrm{C}$

Like previously, the five parameters, $R_{1}, C_{1}, R_{2}, C_{2}$, and $\tau_{1}$, as functions of temperature and dc voltage can be obtained. In this case, the temperature range chosen for the interpolations is between $-20{ }^{\circ} \mathrm{C}$ and $0{ }^{\circ} \mathrm{C}$. Applying the characterization procedure described in Section 5, the five parameters were obtained. Looking at Fig. 7, fitting curves and experimental data show a good agreement for both the capacitance and the resistance versus frequency. This means that the proposed model (2) in the frequency domain is still able to represent the behavior of $\mathrm{LiC}$. 

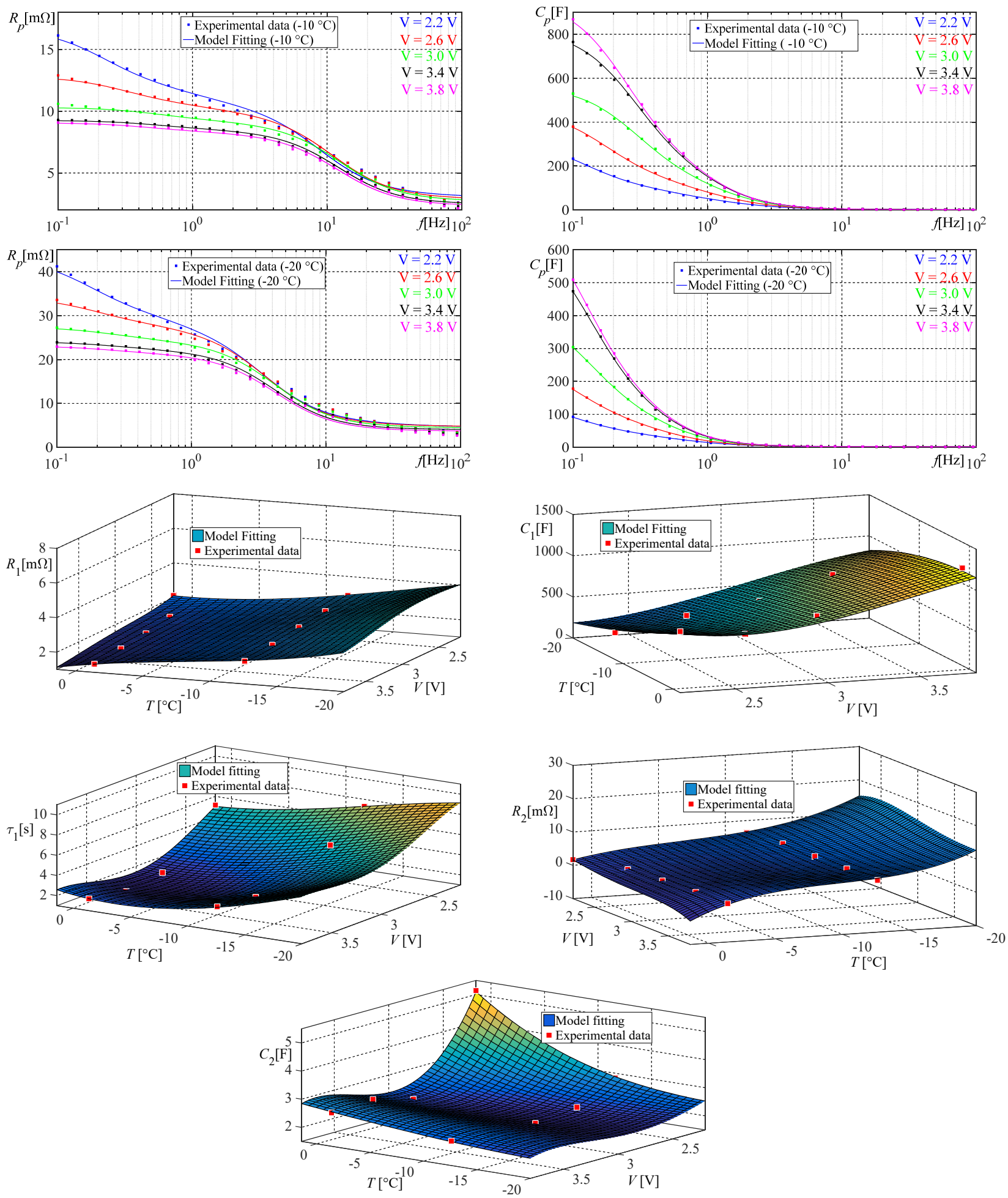

Fig. 7. CLQ1100S1A. Model-based fitting (continuous line) and experimental data (marked point). $-20^{\circ} \mathrm{C}$ and $-10^{\circ} \mathrm{C}$ at different polarization voltages.

In Fig. 7 the values of the five parameters, as functions of temperature and voltage, of the model (2), are reported with their related fitting curves for low temperatures. Table 4 shows the coefficients of the polynomial surfaces related to (4). 


\begin{tabular}{|c|c|c|c|c|c|}
\hline & $R_{1}$ & $C_{1}$ & $\tau_{1}$ & $R_{2}$ & $C_{2}$ \\
\hline$x_{p 00}$ & 0.04069 & 7201 & -152.2 & -0.09557 & 46.23 \\
\hline$x_{p 10}$ & 0.0004658 & 160.1 & 1.394 & 0.006384 & 3.957 \\
\hline$x_{p 01}$ & -0.05009 & -6474 & 253.8 & 0.1646 & -22.42 \\
\hline$x_{p 20}$ & $-6.207 \cdot 10^{-6}$ & 1.855 & -0.04785 & -0.0001848 & 0.05223 \\
\hline$x_{p 11}$ & -0.0005166 & -81.62 & -1.916 & -0.008004 & -3.256 \\
\hline$x_{p 02}$ & 0.02425 & 2021 & -144.8 & -0.09849 & -4.582 \\
\hline$x_{p 30}$ & $7.178 \cdot 10^{-7}$ & - & 0.0005175 & $-3.04 \mathrm{e}-07$ & 0.0002994 \\
\hline$x_{p 21}$ & $1.519 \cdot 10^{-5}$ & -0.6904 & 0.04096 & 0.0001209 & -0.02724 \\
\hline$x_{p 12}$ & 0.0001973 & 10.46 & 0.7678 & 0.00306 & 0.8929 \\
\hline$x_{p 03}$ & -0.005220 & -193.7 & 34.8 & 0.02511 & 3.985 \\
\hline$x_{p 40}$ & - & - & - & - & - \\
\hline$x_{p 31}$ & $-2.561 \cdot 10^{-7}$ & - & $-6.974 \cdot 10^{-5}$ & $-1.157 \cdot 10^{-6}$ & $-8.155 \cdot 10^{-5}$ \\
\hline$x_{p 22}$ & $-4.014 \cdot 10^{-6}$ & - & -0.006055 & $-2.844 \cdot 10^{-5}$ & 0.003583 \\
\hline$x_{p 13}$ & $-2.688 \cdot 10^{-5}$ & - & -0.09221 & -0.0003946 & -0.08084 \\
\hline$x_{p 04}$ & 0.000418 & - & -3.012 & -0.002332 & 0.5313 \\
\hline$x_{p 50}$ & - & - & - & - & - \\
\hline$x_{p 41}$ & - & - & - & - & - \\
\hline$x_{p 32}$ & - & - & - & - & - \\
\hline$x_{p 23}$ & - & - & - & - & - \\
\hline$x_{p 14}$ & - & - & - & - & - \\
\hline
\end{tabular}

In order to test the proposed model at low temperatures in the time domain, similar tests and simulations, as described in Section 6 , were done. The injected current is the same of previous cases.

Fig. 8 shows the comparison of the output voltage trends between experimental data (red line) and simulated ones using the model (2) (blue line), respectively at $-20{ }^{\circ} \mathrm{C}$ and $-10{ }^{\circ} \mathrm{C}$. For these low temperatures, the proposed model is not able to represent the real trend. Even if the frequency trend is well fitted by the proposed model, in the time domain, in particular for high currents, the model is not good at all. From these figures, it is possible to note that the big difference between the experimental and simulated output voltage is due, in particular, to the resistance terms. Therefore, this difference should be due to the different current values. In other words, the resistance terms of model (2) at low temperatures is also depending on the current. More in general, all the parameters could be dependent by the current. But from the figures, it seems that the slopes of the voltage, during charge and discharge, are not changing so much in function of the current and therefore the capacitive behavior seems to be the same. 
In order to support these statements, for these temperatures, the proposed model was corrected by adding the dependence of the resistance terms from the current. In order to do this, from time domain results, the different resistances under the different currents were extracted. In particular, the dc resistance was calculated as the ratio between each current step and the consequent voltage step after the end of the electrical transient. From model (2), it is possible to recognize that the dc resistance, when the frequency tends to zero, is equal to the sum of all resistance terms.

$$
R_{d c}(V, T)=R_{1}(V, T)+R_{2}(V, T)+\sum_{n=1}^{\infty} \frac{2 \tau(V, T)}{n^{2} \pi^{2} C(V, T)}=R_{1}(V, T)+R_{2}(V, T)+\frac{\tau(V, T)}{3 \pi C(V, T)}
$$

This way, the dc resistance should be function of three variables, i.e. voltage, temperature and also current. Since, this interpolation would result very complex to perform, and the aim is to prove the current dependency of the resistance at low temperatures, the dc resistance trend can be obtained as a function only of the current for some fixed temperatures and polarization voltages.

Once the dc resistance trend, at a certain temperature and polarization voltage, is obtained as a function of the current, there is no information about how the single term of the dc resistance is dependent from the current itself. For sake of simplicity, let us suppose that each resistance term of model (2) is current dependent in the same manner. Moreover, the current-resistance trend was expressed in per unit related to the value of dc resistance obtained in the spectroscopy at low frequency.

The interpolations were done using a second degree polynomial function:

$r_{d c}(I)=p_{1} I^{2}+p_{2} I+p_{3}$

Table 5 shows the coefficients of the polynomial functions.

\begin{tabular}{|c|c|c|c|}
\hline & $p_{1}\left[1 / \mathrm{A}^{2}\right]$ & $p_{2}[1 / \mathrm{A}]$ & $p_{3}$ \\
\hline $3.0 \mathrm{~V}-20{ }^{\circ} \mathrm{C}$ & $3.909 \cdot 10^{-5}$ & -0.008174 & 0.7604 \\
\hline $3.0 \mathrm{~V}-10^{\circ} \mathrm{C}$ & $5.226 \cdot 10^{-5}$ & -0.001244 & 1.271 \\
\hline
\end{tabular}

This way, the simulations under $-20{ }^{\circ} \mathrm{C}$ and $-10{ }^{\circ} \mathrm{C}$ show a much better agreement between experimental (red line) and simulation results (green line), as reported in Fig. 8.
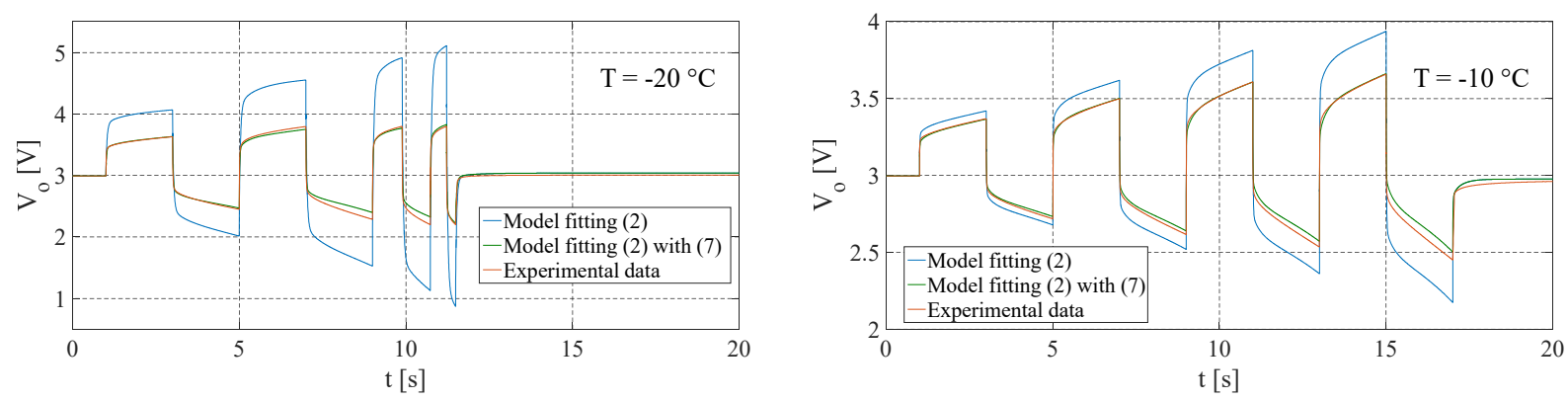

Fig. 8. $3.0 \mathrm{~V}--20^{\circ} \mathrm{C}$ and $-10{ }^{\circ} \mathrm{C}$ with and without current dependency 
Of course, the proposed model may be better improved by adding the current dependency as a further variable, besides voltage and temperature, in the resistance terms and also in the other parameters. In order to do this, we need to interpolate each parameter of model (2) as functions of the three variables namely voltage, temperature and current. This means to perform, besides the tests at different polarization voltages and temperatures, other tests also at different dc rated currents to extract the behavior of parameters as a function of current as well. Nevertheless, as already claimed in Section 2, the use of GEIS technique with adding dc currents provokes a change in the polarization voltage making the use of this technique impossible. Consequently, it is better to use some other techniques based on time domain analysis to obtain the current dependency, as done in this case, for the currentresistance trend. However, by these simulations, it is possible to confirm that, at low temperatures, the current causes a change in resistance terms more than in the capacitance ones.

\section{Conclusion}

Lithium ion capacitors are spreading in recent years because they present a power density almost comparable with that of electric double layer capacitors but with an increased energy density. Therefore, they represent a good solution to realize storage systems whose characteristics are between LiBs and ELDCs. Their main limit, compared with ELDCs, is in their degraded performances at low temperatures. For this reason, particular investigation on their use at low temperatures can be very interesting.

The spreading of LiCs leads to an increasing attention for their modeling. Consequently, in recent years, some models of LiCs, sometimes taken from ELDCs, have been proposed in literature. Anyway, electric equivalent circuits, capable of taking into account also the behavior of LiCs in a wide temperature range, has been provided but they are very complicated, with a lot of parameters to be estimated. In this paper, a simple model, valid at fixed temperature, has been extended to obtain a model capable of representing $\mathrm{LiC}$ behavior from $0^{\circ} \mathrm{C}$ to $60^{\circ} \mathrm{C}$. The proposed model can be summarized in an equivalent circuit with five independent parameters. These parameters are variable in voltage and temperature and, starting from experimental results, their variation has been obtained by means of some polynomial surface interpolations. Moreover, in the paper, some additional experimental tests, in time domain, have been performed in order to verify the goodness of the model. The results reported in the paper show that, in all voltage ranges and for temperature between $0^{\circ} \mathrm{C}$ and $60{ }^{\circ} \mathrm{C}$, the proposed model is able to represent, with a very good accuracy, the behavior of LiCs by neglecting the current effect. On other hands, for temperatures between $-20^{\circ} \mathrm{C}$ and 0 ${ }^{\circ} \mathrm{C}$, the proposed model is not able to represent the behavior of LiCs because the effect of different currents becomes important at these temperatures. Indeed, for low temperatures, the spectroscopies show a LiC behavior very similar to that of LiBs. Consequently, from $60{ }^{\circ} \mathrm{C}$ to $0^{\circ} \mathrm{C}$ the current effect can be neglected. At $0{ }^{\circ} \mathrm{C}$ this effect starts to arise. For temperatures lower than $0{ }^{\circ} \mathrm{C}$ the current effect cannot be neglected, in particular, for the resistance terms. In the paper, it has been shown that, if the 
variation of the resistance with the current is taken into account, the proposed model gives very good results also at very low temperatures. 


\section{References}

[1] S.S Williamson, A.K. Rathore, F. Musavi, Industrial Electronics for Electric Transportation: Current State-of-the-Art and Future Challenges, IEEE Transactions on Industrial Electronics, vol.62, no.5, pp.3021-3032, May 2015

[2] B.K. Bose, Global Energy Scenario and Impact of Power Electronics in 21st Century, IEEE Transactions on Industrial Electronics, vol.60, no.7, pp.26382651, July 2013

[3] Aurelien Du Pasquier, Irene Plitz, Serafin Menocal, Glenn Amatucci, A comparative study of Li-ion battery, supercapacitor and nonaqueous asymmetric hybrid devices for automotive applications, Journal of Power Sources, Volume 115, Issue 1, 27 March 2003, Pages 171-178, ISSN 0378-7753, http://dx.doi.org/10.1016/S0378-7753(02)00718-8.

[4] S. Grillo, V. Musolino, L. Piegari, E. Tironi, C. Tornelli, DC Islands in AC Smart Grids, IEEE Transactions on Power Electronics, vol.29, no.1, pp.89-98, Jan. 2014

[5] V.A. Boicea, Energy Storage Technologies: The Past and the Present, in Proceedings of the IEEE, vol.102, no.11, pp.1777-1794, Nov. 2014

[6] M.D. Mufti, S.J. Iqbal, S.A. Lone, Qurat-ul Ain, Supervisory Adaptive Predictive Control Scheme for Supercapacitor Energy Storage System, IEEE Systems Journal, vol.9, no.3, pp.1020-1030, Sept. 2015

[7] J. Pegueroles-Queralt, F.D. Bianchi, O. Gomis-Bellmunt, A Power Smoothing System Based on Supercapacitors for Renewable Distributed Generation, IEEE Transactions on Industrial Electronics, vol.62, no.1, pp.343-350, Jan. 2015

[8] Ding Wu; R. Todd, A.J. Forsyth, Adaptive Rate-Limit Control for Energy Storage Systems, IEEE Transactions on Industrial Electronics, vol.62, no.7, pp.4231-4240, July 2015

[9] N. Omar, M. Al Sakka, J. Van Mierlo, P. Van den Bossche, H. Gualous, Electric and thermal characterization of advanced hybrid Li-Ion capacitor rechargeable energy storage system, 2013 International Conference on Power Engineering, Energy and Electrical Drives (POWERENG), pp.1574-1580, 13-17 May 2013

[10] Cheng Zhang, Kang Li, S. McLoone, Zhile Yang, Battery modelling methods for electric vehicles - A review, 2014 European Control Conference (ECC), pp.2673-2678, 24-27 June 2014

[11] S.A. Hamidi, E. Manla, A. Nasiri, Li-ion batteries and Li-ion ultracapacitors: Characteristics, modeling and grid applications, 2015 IEEE Energy Conversion Congress and Exposition (ECCE), pp.4973-4979, 20-24 Sept. 2015

[12] Y. Firouz, N. Omar, P. Van den Bossche, J. Van Mierlo, Electro-Thermal Modeling of New Prismatic Lithium-Ion Capacitors, 2014 IEEE Vehicle Power and Propulsion Conference (VPPC), pp.1-6, 27-30 Oct. 2014

[13] S. Barcellona, F. Ciccarelli, D. Iannuzzi, L. Piegari, Modeling and Parameter Identification of Lithium-Ion Capacitor Modules, IEEE Transactions on Sustainable Energy, , vol.5, no.3, pp.785-794, July 2014

[14] Patricia H. Smith, Thanh N. Tran, Thomas L. Jiang, Jaesik Chung, Lithium-ion capacitors: Electrochemical performance and thermal behavior, Journal of Power Sources, Volume 243, 1 December 2013, Pages 982-992, ISSN 0378-7753, http://dx.doi.org/10.1016/j.jpowsour.2013.06.012.

[15] Y. Firouz, N. Omar, J.-M. Timmermans, P. Van den Bossche, J. Van Mierlo, Lithium-ion capacitor - Characterization and development of new electrical model, Energy, Volume 83, 1 April 2015, Pages 597-613, ISSN 0360-5442, http://dx.doi.org/10.1016/j.energy.2015.02.069. 
[16] E. Manla, G. Mandic and A. Nasiri, Development of an Electrical Model for Lithium-Ion Ultracapacitors, in IEEE Journal of Emerging and Selected Topics in Power Electronics, vol. 3, no. 2, pp. 395-404, June 2015

[17] Bromwich TJIA. An introduction to the theory of infinite series. 2nd ed. Macmillan and Co..; 1931

[18] V. Musolino, L. Piegari, E. Tironi, New Full-Frequency-Range Supercapacitor Model With Easy Identification Procedure, IEEE Transactions on Industrial Electronics, vol.60, no.1, pp.112-120, Jan. 2013.

[19] V. Khomenko, E. Raymundo-Piñero, F. Béguin, High-energy density graphite/AC capacitor in organic electrolyte, Journal of Power Sources, Volume 177, Issue 2, 1 March 2008, Pages 643-651, ISSN 0378-7753, http://dx.doi.org/10.1016/j.jpowsour.2007.11.101.

[20] D. Andre, M. Meiler, K. Steiner, H. Walz, T. Soczka-Guth, D.U. Sauer, Characterization of high-power lithium-ion batteries by electrochemical impedance spectroscopy. II: Modelling, Journal of Power Sources, Volume 196, Issue 12, 15 June 2011, Pages 5349-5356, ISSN 0378-7753, http://dx.doi.org/10.1016/j.jpowsour.2010.07.071.

[21] G. Fan, K. Pan and M. Canova, A comparison of model order reduction techniques for electrochemical characterization of Lithium-ion batteries, IEEE Conference on Decision and Control (CDC), Osaka, 2015, pp. 3922-3931. 